\title{
Correction to: Molecular constitutive equation for unentangled branch copolymers
}

\author{
Zhongqiang Xiong ${ }^{1} \cdot$ Wei Yu ${ }^{1}$
}

Published online: 31 January 2022

○) Springer-Verlag GmbH Germany, part of Springer Nature 2022

Correction to: Rheologica Acta (2021) 60:439-455

https://doi.org/10.1007/s00397-021-01273-5

In this paper, a mistake in Eq. 21, which does not impact other conclusions, must be corrected:

From the definition of diffusion coefficient, $D$, by using the normal coordinates (Eqs. 29 and 33 in Appendix 2), one obtains,

$D=\lim _{t \rightarrow \infty} \frac{1}{6 t}\left\langle\left[\boldsymbol{R}_{c}(t)-\boldsymbol{R}_{c}(0)\right]^{2}\right\rangle=\frac{1}{n_{b}^{2}} \sum_{u=1}^{n_{b}} \sum_{v=1}^{n_{b}} k_{B} T \alpha_{u p^{*}} \alpha_{v p^{*}}$

$D=\frac{k_{B} T}{\operatorname{tr}(\zeta)}$

where $\alpha_{u p^{*}}$ is obtained from the eigenvector corresponding to the zero eigenvalue $\lambda_{p^{*}}=0$, in the absence of external flow field and confinements $(\boldsymbol{K}=0$ and $\overline{\boldsymbol{K}}=0)$.

The original article has been corrected.

Publisher's note Springer Nature remains neutral with regard to jurisdictional claims in published maps and institutional affiliations.

The original article can be found online at https://doi.org/10.1007/ s00397-021-01273-5.

Wei Yu

wyu@sjtu.edu.cn

1 Advanced Rheology Institute, Department of Polymer

Science and Engineering, Frontiers Science Center for Transformative Molecules, State Key Laboratory for Metal Matrix Composite Materials, Shanghai Jiao Tong

University, Shanghai 200240, People's Republic of China 